\title{
A density functional study of vacancy formation in grain boundaries of undoped $\alpha$-alumina
}

\author{
Takafumi Ogawa ${ }^{a, *}$, Akihide Kuwabara ${ }^{a}$, Craig A. J. Fisher ${ }^{a}$, Hiroki \\ Moriwake $^{\mathrm{a}}$, Katsuyuki Matsunaga ${ }^{\mathrm{b}}$, Kenji Tsuruta ${ }^{\mathrm{c}}$, Satoshi Kitaoka ${ }^{\mathrm{d}}$ \\ ${ }^{a}$ Nanostructures Research Laboratory, Japan Fine Ceramics Center, 2-4-1 Mutsuno, \\ Atsuta-ku, Nagoya 456-8587, Japan \\ ${ }^{b}$ Department of Materials Science and Engineering, Nagoya University, Furo-cho, \\ Chikusa-ku, Nagoya 464-8603, Japan \\ ${ }^{c}$ Graduate School of Natural Science and Technology, Okayama University, 3-1-1 \\ Tsushima-naka, Kita-ku, Okayama 700-8530, Japan \\ ${ }^{d}$ Materials Research and Development Laboratory, Japan Fine Ceramics Center, 2-4-1 \\ Mutsuno, Atsuta-ku, Nagoya 456-8587, Japan
}

\section{Abstract}

Recent oxygen permeation experiments of $\alpha$-alumina have shown that the major diffusion species at grain boundaries, i.e., oxygen and aluminum vacancies, switch over depending on the oxygen partial pressures at high temperatures, namely appearance of the $p-n$ transition. In order to investigate the transition behavior, formation energies of charged oxygen and aluminum vacancies at $\mathrm{Al}_{2} \mathrm{O}_{3}$ grain boundaries are evaluated by using density functional theory (DFT). The electronic structures affecting the carrier concentrations are assessed for three types of grain boundaries. The ratios of the grain boundary band gaps relative to the bulk value lie in the range of $0.6-0.85$ depending on the degree of grain boundary misorientations. From the formation energies we find that, at band gaps around $60 \%$ compared to the

\footnotetext{
*Corresponding author. Tel.: +81 52871 3500; fax: +81 528713599.

Email address: t_ogawa@jfcc.or.jp (Takafumi Ogawa)
} 
bulk value, the dominant vacancy alters depending on the pressure, consistent with the oxygen permeation experiments. Our results suggest that the band bap narrowing occurring at grain boundaries enhances the creation of oxygen vacancies and conduction electrons, facilitating the observed transition.

Keywords: Alumina, Density functional theory, Vacancies, Grain-boundary diffusion, Oxygen permeation

\section{Introduction}

Grain boundary (GB) diffusion of intrinsic species in $\alpha$-alumina is a fundamental and significant phenomenon affecting diffusional creep, sintering processes, and oxidation of alumina-scale-forming alloys, and thus has an important effect on its performance in various applications. In thermal barrier coating systems, the $\alpha$-alumina scale formed on alumina-forming alloys provides protection against further oxidation, contributing to an increase in the durability of the system at high temperatures $[1,2]$. In this context, it is important to control the GB diffusion by addition of reactive elements such as $\mathrm{Y}, \mathrm{Lu}$, and Hf, which segregate to GBs. There are, however, unresolved issues concerning GB diffusion in $\alpha$-alumina even in the undoped case. In this paper, we provide a theoretical basis for understanding the GB diffusion behavior observed in oxygen permeation experiments of undoped $\alpha$-alumina $[3,4]$ which were designed to investigate the oxidation process.

In a series of oxygen permeation experiments $[3,4,5,6,7,8]$, different oxygen partial pressures imposed on two sides of a sample wafer, labeled $P_{\mathrm{O}_{2}}^{\text {high }}$ and $P_{\mathrm{O}_{2}}^{\text {low }}$, induced oxygen permeation at high temperatures from $1750 \mathrm{~K}$ to 
1950 K. Observed surface morphologies around GBs after the permeation experiments and the associated measured permeabilities were interpreted in terms of two regimes. When $P_{\mathrm{O}_{2}}^{\text {high }}=1 \mathrm{~Pa}$ and $P_{\mathrm{O}_{2}}^{\text {low }}=10^{-8}-10^{-4} \mathrm{~Pa}$, oxygen vacancies formed on the $P_{\mathrm{O}_{2}}^{\text {low }}$ side through release of oxygen molecules diffuse to the opposite $\left(P_{\mathrm{O}_{2}}^{\text {high }}\right)$ side and contribute to the dissociative reaction of oxygen molecules on that side. The overall reaction can be expressed in Kröger-Vink notation as

$$
\frac{1}{2} \mathrm{O}_{2}+V_{\mathrm{O}}^{\bullet \bullet}+2 \mathrm{e}^{\prime} \leftrightarrow \mathrm{O}_{\mathrm{O}}^{\times}
$$

where $V$ is a vacancy and $\mathrm{e}^{\prime}$ is an electron. On the other hand, when $P_{\mathrm{O}_{2}}^{\text {low }}=$ $1 \mathrm{~Pa}$ and $P_{\mathrm{O}_{2}}^{\text {high }}=10^{3}-10^{5} \mathrm{~Pa}$, aluminum vacancies are created on the $P_{\mathrm{O}_{2}}^{\text {high }}$ side through an alumina-forming reaction expressed by

$$
\frac{1}{2} \mathrm{O}_{2} \rightarrow \mathrm{O}_{\mathrm{O}}^{\times}+\frac{2}{3} V_{\mathrm{Al}}^{\prime \prime \prime}+2 \mathrm{~h}^{\bullet}
$$

where $\mathrm{h}^{\bullet}$ is a hole, and diffuse to the $P_{\mathrm{O}_{2}}^{\text {low }}$ side where the opposite reaction to Eq. (2) occurs, yielding oxygen molecules. GB diffusion coefficients, $D_{\mathrm{gb}}$, of $\mathrm{O}$ and $\mathrm{Al}$ were calculated from the permeation measurements under the limited $P_{\mathrm{O}_{2}}$ range. These two different regimes indicate that the diffusing species in GBs alter as a function of $\Delta P_{\mathrm{O}_{2}}$. This switchover has also been observed in oxygen permeation measurements of Hf-, Lu-, and Y-doped alumina, although the diffusivities are partially suppressed $[5,6,8]$. In order to understand the suppression mechanism, it is first necessary to understand the behavior in undoped samples. Although tracer experiments of oxygen GB diffusion have been performed $[9,10,11,12,13]$, the dependence on $P_{\mathrm{O}_{2}}$ is inaccessible and reports of tracer diffusion of aluminum have been limited to bulk crystals due to experimental difficulties $[14,15]$. 
Heuer et al. [16] pointed out the importance of electronic structures for interpretation of the switchover of the GB-diffusion species, and referred to the phenomenon induced by $P_{\mathrm{O}_{2}}$ as the $p-n$ transition, since the dominant electronic carriers are considered to switch between the two regimes as a result of the reactions in Eqs. (1) and (2). As discussed in their paper, density functional theory (DFT) calculations of point defect formation energies in $\alpha$-alumina $[17,18,19,20]$ are useful to investigate how electronic structures affect the defect stabilities and GB diffusion. In DFT calculations, following the widely used formulation proposed by Zhang and Northrup [21], defect formation energies and related defect concentrations are defined as a function of oxygen partial pressure and temperature through oxygen chemical potentials, $\mu_{\mathrm{O}_{2}}$, as well as electron chemical potentials, $\mu_{\mathrm{e}}$. Because migration barriers are independent of $P_{\mathrm{O}_{2}}$, the switchover depends only on the formation energies. To determine the energies using those theoretical calculations, $\mu_{\mathrm{e}}$ must be determined. Although Hine et al. have shown calculation of $\mu_{\mathrm{e}}$ for the Schottky pair [19], reports including contributions of conduction electrons and holes have yet to be found, required to gauge the effect of electronic structures quantitatively.

In this paper, we investigate the $P_{\mathrm{O}_{2}}$ dependence of defect formation energies in balance with electronic carriers based on first-principles DFT. Here, we limit our discussion to the dominant defect types, fully charged oxygen and aluminum vacancies, i.e., $V_{\mathrm{O}}^{\bullet \bullet}$ and $V_{\mathrm{Al}}^{\prime \prime \prime}$ for clarity and simplicity $[17,18]$. In order to incorporate the electronic character of GBs, we examine electronic structures of two types of $\Sigma 13$ GBs $[22,23,24]$ and a $\Sigma 31$ GB $[25,26]$ whose structures in bicrystal samples have been characterized by 
high-resolution transmission electron microscopy (HRTEM) and theoretical calculations (including DFT) based on coincident site lattice (CSL) theory.

\section{Methodology}

\subsection{Formation energies}

The formation energies of vacancies in $\alpha-\mathrm{Al}_{2} \mathrm{O}_{3}$ denoted by $V_{\eta}^{q}$, where $\eta$ is whether $\mathrm{O}$ or $\mathrm{Al}$ and $q$ is the charge state, are given by [21]

$$
E_{\mathrm{f}}^{V_{\eta}^{q}}=E_{\mathrm{T}}^{V_{\eta}^{q}}-E_{\mathrm{T}}^{\text {Pristine }}+\mu_{\eta}\left(P_{\mathrm{O}_{2}}, T\right)+q \mu_{\mathrm{e}}
$$

Here, $E_{\mathrm{T}}^{V_{\eta}^{q}}$ and $E_{\mathrm{T}}^{\text {Pristine }}$ are the calculated total energies of the vacancy and pristine systems, respectively, and $\mu_{\eta}$ and $\mu_{\mathrm{e}}$ are the chemical potentials of $\eta$ atoms and electrons, respectively. The atomic chemical potentials are determined by the procedure of Hine et al. [18]. When in equilibrium with the surrounding gas, by equating the oxygen chemical potential with half the chemical potential of $\mathrm{O}_{2}$ gas, i.e., $\frac{1}{2} \mu_{\mathrm{O}_{2}(g)}$, the formation energies can be connected with temperature and oxygen pressure using the Gibbs free energy of $\mathrm{O}_{2}$ gas which is calculated using thermochemical data [27] within the ideal gas approximation. The chemical potential under standard conditions, $\mu_{\mathrm{O}_{2}}^{0}$, can be obtained from

$$
\Delta G_{\mathrm{f}\left(\mathrm{Al}_{2} \mathrm{O}_{3}\right)}^{0}=E_{\mathrm{Al}_{2} \mathrm{O}_{3}}-2 E_{\mathrm{Al}(\mathrm{s})}-\frac{3}{2} \mu_{\mathrm{O}_{2}}^{0}
$$

Here, $E_{\mathrm{Al}_{2} \mathrm{O}_{3}}$ and $E_{\mathrm{Al}(\mathrm{s})}$ are the DFT energies of the bulk alumina and metal aluminum per formula unit, respectively, which are approximately equal to the Gibbs free energies under standard conditions. The formation energy of $\alpha$-alumina, $\Delta G_{\mathrm{f}\left(\mathrm{Al}_{2} \mathrm{O}_{3}\right)}^{0}$, is also taken from experimental results [27]. Under 
equilibrium conditions, $\mu_{\mathrm{Al}}$ is given through $E_{\mathrm{Al}_{2} \mathrm{O}_{3}}=2 \mu_{\mathrm{Al}}+3 \mu_{\mathrm{O}}$ by neglecting the vibrational contributions to the Gibbs free energy. $\mu_{\mathrm{e}}$ is measured with respect to the valence band maximum (VBM), i.e., $\mu_{\mathrm{e}}=\varepsilon_{\mathrm{F}}+\varepsilon_{\mathrm{VBM}}$, where $\varepsilon_{\mathrm{F}}$ is the Fermi level. Thus, the formation energies of oxygen and aluminum vacancies are rewritten as

$$
E_{\mathrm{f}}^{V \ddot{\circ}}=E_{0}^{V \ddot{\circ}}+\Delta \mu_{\mathrm{O}}\left(T, P_{\mathrm{O}_{2}}\right)+2 \varepsilon_{\mathrm{F}}
$$

and

$$
E_{\mathrm{f}}^{V_{\mathrm{Al}}^{\prime \prime \prime}}=E_{0}^{V_{\mathrm{Al}}^{\prime \prime \prime}}-\frac{2}{3} \Delta \mu_{\mathrm{O}}\left(T, P_{\mathrm{O}_{2}}\right)-3 \varepsilon_{\mathrm{F}}
$$

where $\Delta \mu_{\mathrm{O}}$ is the oxygen chemical potential with respect to the value at $T=2371 \mathrm{~K}$ and $P_{\mathrm{O}_{2}}=0.2$ atm. $E_{0}$ was obtained from previous DFT calculations $[18]$.

In order to determine the Fermi level, we impose the charge neutrality condition given by

$$
2\left[V_{\mathrm{O}}^{\bullet \bullet}\right]+\left[\mathrm{h}^{\bullet}\right]=3\left[V_{\mathrm{Al}}^{\prime \prime \prime}\right]+\left[\mathrm{e}^{\prime}\right]
$$

The concentration of vacancies, $\left[V_{\eta}^{q}\right]$, was calculated according to

$$
\left[V_{\eta}^{q}\right]=N_{\eta} \exp \left(-E_{\mathrm{f}}^{V_{\eta}^{q}} / k_{\mathrm{B}} T\right)
$$

where $N_{\eta}$ are the concentrations of $\eta$ lattice sites per volume and the vibrational entropy terms are ignored. The hole and conduction electron densities are evaluated through

$$
\left[\mathrm{h}^{\bullet}\right]=N_{\mathrm{v}} \exp \left(-\varepsilon_{\mathrm{F}} / k_{\mathrm{B}} T\right)
$$

and

$$
\left[\mathrm{e}^{\prime}\right]=N_{\mathrm{c}} \exp \left(-\left(E_{\mathrm{g}}-\varepsilon_{\mathrm{F}}\right) / k_{\mathrm{B}} T\right),
$$


where $E_{\mathrm{g}}$ is the band gap and $N_{\mathrm{v}}$ and $N_{\mathrm{c}}$ are the effective densities of states of valence and conduction bands, respectively. The usage of these formula is validated when the numerator of the exponential is sufficiently larger than $k_{\mathrm{B}} T \approx 0.17 \mathrm{eV}$ for $T=2000 \mathrm{~K}$ and this is satisfied in our cases due to the wide band gaps of $\alpha$-alumina crystals. $N_{\mathrm{v}}$ and $N_{\mathrm{c}}$ are calculated from $2\left(m_{\mathrm{h}} k_{\mathrm{B}} T / 2 \pi \hbar^{2}\right)^{3 / 2}$ and $2\left(m_{\mathrm{e}} k_{\mathrm{B}} T / 2 \pi \hbar^{2}\right)^{3 / 2}$, respectively, where $m_{\mathrm{h}}$ and $m_{\mathrm{e}}$ are the effective masses of holes and electrons, respectively, which we calculated by DFT. Here, the multiplicity factors are taken to be unity because the bottom of the conduction band and the top of the valence band of $\alpha$ alumina are situated at the $\Gamma$ point of the Brillouin zone, as shown in Fig. 2 .

\subsection{DFT calculation details}

In DFT calculations, it is well known that the conventional local density approximation (LDA) and general gradient approximation (GGA) for the exchange-correlation functional usually underestimate band gaps in the case of insulators and semiconductors. Because this problem has a large effect on formation energies of defects with occupied states in the band gap, empirical corrections for the band gap, e.g., ridged shift of the conduction band and defect levels in the middle of the band gap to adjust the gap to the experimental value, have been adopted in LDA and GGA studies of intrinsic point defects in $\alpha$-alumina $[17,18]$. Recently, hybrid density functionals combining LDA or GGA functionals and non-local Fock exchange components have been developed to obtain more accurate band gaps, and thus defect formation energies, resulting in a revision of thermal transition levels in $\alpha$-alumina [20]. It is not possible to compare these data directly, however, because the 
correction methods for the Coulomb interaction between periodic images in charged defect calculations are different. In this work, our interest is in the

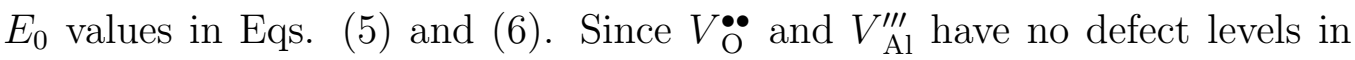
the band gap, the difference in $E_{0}$ between the functionals is expected to be slight, so we adopt the LDA values for $E_{0}$ in this study [18].

To obtain electronic structures of bulk and GB systems, DFT calculations have carried out the projector augmented wave (PAW) method [28] based on plane-wave bases as implemented in VASP [29]. For the exchange-correlation functional, the Perdew-Burke-Ernzerhof generalized gradient approximation (PBE-GGA) [30] and HSE06 (after Heyd, Scuseria and Ernzerhof) hybridfunctional [31] were used. A plane-wave cutoff energy of $500 \mathrm{eV}$ was used and the Brillouin zone (BZ) was sampled by the Monkhorst-Pack method [32]. The residual atomic forces in the relaxed structures were lower than $0.01 \mathrm{eV} / \AA$. For bulk $\alpha$-alumina, the rhombohedral cell was relaxed using a $5 \times 5 \times 5 k$-point grid. The effective masses were calculated by fitting parabola around the valence band maximum and conduction band minimum.

The preparation of the $\Sigma 13$ and $\Sigma 31$ GB models follows previous reports. The $\Sigma 13$ tilt GB was constructed by rotating one half-crystal by $103.6^{\circ}$ around the $[10 \overline{1} 0]$ axis. From all possible $\Sigma 13$ (1010) GBs, two Alterminated and O-terminated configurations, labeled $\mathrm{G}(\mathrm{Al})$ and $\mathrm{G}(\mathrm{O})$ [22], were selected. The structures are shown in Fig. 1 (a) and (b). The two GBs in each cell, which contain 120 atoms, are separated by about $15.5 \AA$. A $\Sigma 31$ tilt GB was constructed by rotating one half-crystal by $17.9^{\circ}$ around the [0001] axis. We used the structural model shown in Fig. 1 (c), as it reproduces the atomic arrangement observed by HRTEM [25, 26]. This contains 
620 atoms in the cell with GBs separated by about $13.8 \AA$ A. For BZ sampling, a $3 \times 3 \times 1 k$-point grid and $\Gamma$ point only were used for the $\Sigma 13$ and $\Sigma 31$ GBs, respectively. The GB structures obtained by relaxing with the GGA functional were used in HSE06 calculations to minimize the computational cost.

\section{Results and discussion}

\subsection{Electronic structures of bulk and grain boundaries}

The lattice constants of relaxed bulk structures of rhombohedral $\alpha-\mathrm{Al}_{2} \mathrm{O}_{3}$ were $a=5.178 \AA$ and $\alpha=55.30^{\circ}$ for GGA and $a=5.122 \AA$ and $\alpha=55.33^{\circ}$ for HSE06, which are close to experimental values of $a=5.136$ and $\alpha=$ $55.28^{\circ}[33]$. This may indicate that the trend in GGA to overestimate lattice constants is improved by use of the HSE06 hybrid functional. The band structures obtained from GGA and HSE06 calculations are shown in Fig. 2 (a) and (b), respectively. The band profiles are similar to each other except for the energy levels of the conduction bands. By virtue of hybridizing the Fock exchange potential, the bulk band gap according to HSE06, $E_{\mathrm{g}}^{\mathrm{B}}=8.16 \mathrm{eV}$, is closer to the experimental values of 8.8 to $9.2 \mathrm{eV}[34,35]$ than $E_{\mathrm{g}}^{\mathrm{B}}=5.87 \mathrm{eV}$ according to GGA. The calculated effective masses show a large anisotropy only for the valence band maximum, as shown in the inset of Fig. 2 (b). This is because of bands that exist about $0.1 \mathrm{eV}$ below the maximum at the $\Gamma$ point. We assume a prolate spheroidal shape whose longer axis is in the [111] direction (corresponding to the [0001] direction in the hexagonal representation) and whose shorter axes are in the [1ํㅣㄹ and equivalent orthonor-

mal directions. While GGA gives $m_{\mathrm{h}}^{[111]} / m_{0}=0.70$ and $m_{\mathrm{h}}^{[1 \overline{1} 0]} / m_{0}=15.08$, 
HSE06 gives $m_{\mathrm{h}}^{[111]} / m_{0}=0.49$ and $m_{\mathrm{h}}^{[11 \overline{1} 0]} / m_{0}=10.46$. Average effective masses for the effective density of states, $\left(m_{\mathrm{h}}^{[111]} m_{\mathrm{h}}^{[1 \overline{1} 0]} m_{\mathrm{h}}^{[1 \overline{1} 0]}\right)^{1 / 3}$, are $5.42 m_{0}$ for GGA and $3.77 m_{0}$ for HSE06. On the other hand, the minimum of the conduction band shows almost isotropic behavior. The differences between $m_{\mathrm{h}}^{[111]} / m_{0}$ and $m_{\mathrm{h}}^{[1 \overline{0} 0]} / m_{0}$ are less than 0.1. The average effective masses are 0.74 for GGA and 0.75 for HSE06, being independent of the functional used. Although here we use the values of HSE06 for the concentrations of carriers, our conclusions are unaltered by the use of values obtained using GGA.

Figure 3 shows the density of states (DOS) of the three types of GBs according to HSE06. While the valence bands of the bulk and $\Sigma 13 \mathrm{G}(\mathrm{Al})$ GB are similar to each other, the highest occupied level of the $\Sigma 13 \mathrm{G}(\mathrm{O})$ and $\Sigma 31$ GBs are isolated. The latter are localized states at the GBs as shown in the example of Fig. 4, in which the isosurface of the partial charge density corresponding to the peak in the DOS of the $\Sigma 13 \mathrm{G}(\mathrm{O}) \mathrm{GB}$ is depicted. At the $\Sigma 13 \mathrm{G}(\mathrm{O}) \mathrm{GB}$, nearest-neighboring oxygen atoms at the GB plane are coordinated by three aluminum atoms, less than the four atoms of other oxygen atoms at the GB and in the bulk-like region. Figure 4 shows that those oxygen atoms with dangling bonds contribute the localized states in Fig. 3 (c). This localized character has been seen in a few GBs in $\mathrm{ZnO}[36]$. A similar isolated peak is also found in the $\Sigma 31$ GB DOS (Fig. 3 (d)). The conduction bands of the GBs are strongly influenced by their atomic structures, shifting to a lower energy range compared to the bulk, as seen in Fig. 3. These changes induce a narrowing of the band gap.

The band gaps of the GBs relative to that of the bulk crystal are plotted in Fig. 5 as a function of the grain boundary energy, $E_{\mathrm{GB}}$, for both function- 
als. This figure shows that GB band gaps correlate well with the degree of interface coincidence despite the difference in the relative band gaps, $E_{\mathrm{g}} / E_{\mathrm{g}}^{\mathrm{B}}$, and $E_{\mathrm{GB}}$ between the functionals. In HSE06 calculations, the relative band gap of the $\Sigma 31 \mathrm{~GB}$ reaches around $60 \%$, so that this drastic change must affect the defect creation through the electron formation described by Eq. (10). Because the relative band gap of amorphous $\mathrm{Al}_{2} \mathrm{O}_{3}$ by LDA has a similar value [37] (also shown in Fig. 5), the narrowing of the band gap may be limited to around $60 \%$.

Oxygen permeation experiments have been carried out to measure aluminum diffusion using alumina bicrystals forming $\Sigma 13$ and $\Sigma 31$ GBs [7]. According to these measurements, while the migration in the $\Sigma 13$ GB bicrystal was too small to be observed, $\mathrm{Al}$ diffusion coefficients at the $\Sigma 31 \mathrm{~GB}$ were comparable to that in polycrystalline alumina. For the $\Sigma 31 \mathrm{~GB}$, oxygen GB diffusion coefficients measured by tracer experiments [13] have also proven to be comparable to those of polycrystalline $\mathrm{Al}_{2} \mathrm{O}_{3}$ reported in the literature [9]. These bicrystal experiments showed that GB diffusivity is closely related to the atomic structures of the interface. Our calculations indicate that the band gap of a GB depends on the interface atomic structure, which will thus affect the defect formation energetics at GBs, as described in the next section.

Experiments based on spatially resolved valence electron energy-loss spec-

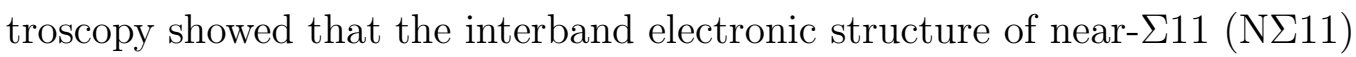
GB exhibits increased ionicity around the GB, suggesting a reduction of the band gap, although this could not be quantified [38]. First principles calculations of $\mathrm{N} \Sigma 11$ GB revealed a low $E_{\mathrm{GB}}$ of $1.7 \mathrm{~J} / \mathrm{m}^{2}$ and a band gap reduction 
of $1.0 \mathrm{eV}$ from a bulk gap of about $6.0 \mathrm{eV}$ to give a relative band gap of 0.83 [39]. These values are similar to those for a $\Sigma 13 \mathrm{G}(\mathrm{Al}) \mathrm{GB}$ in Fig. 5. The observation that no levels are introduced into the band gap in the N 211 GB is also consistent with the results for $\Sigma 13 \mathrm{G}(\mathrm{Al}) \mathrm{GB}$ in Fig. 3 (b). These results indicate that the $\mathrm{N} \Sigma 11 \mathrm{~GB}$ is relatively coherent, similar to the $\Sigma 13 \mathrm{G}(\mathrm{Al})$ GB.

The temperature dependence of band gaps, which is approximated as a proportional relation at high temperatures, should be considered because the temperature-induced band gap change in $\alpha-\mathrm{Al}_{2} \mathrm{O}_{3}$ is large compared to that of typical semiconductors such as Si and Ge [34]. Experiments of interband electronic structures by vacuum ultraviolet spectroscopy over a wide temperature range from $293 \mathrm{~K}$ up to $2167 \mathrm{~K}$ gave a linear coefficient at high temperatures of $-0.85 \mathrm{meV} / \mathrm{K}[40]$, which is much larger than those for $\mathrm{Si}$ $(-0.22 \mathrm{meV} / \mathrm{K})$ or $\mathrm{Ge}(-0.44 \mathrm{meV} / \mathrm{K})[41]$. Using this value for the coefficient and $E_{\mathrm{g}}^{\mathrm{B}}=9.1 \mathrm{eV}$ for the bulk band gap at $0 \mathrm{~K}$, the gap at $1900 \mathrm{~K}$ is reduced to $7.49 \mathrm{eV}$ or $82 \%$. Although this suggests that GB band gaps will be further reduced from the calculated values at $0 \mathrm{~K}$, the temperature dependence of a GB's band gap will be different from that of the bulk crystal and consequently difficult to predict. In this work, we consider the relative band gap to be a variable in the range of $0.5<E_{\mathrm{g}} / E_{\mathrm{g}}^{\mathrm{B}}<1.0$.

\subsection{Formation energies}

Formation energies of $\mathrm{O}$ and $\mathrm{Al}$ vacancies at $1900 \mathrm{~K}$ were calculated from DFT results after determining $\varepsilon_{\mathrm{F}}$. In the calculations, the experimental value of $9.1 \mathrm{eV}$ for $E_{\mathrm{g}}^{\mathrm{B}}$ was used for a $P_{\mathrm{O}_{2}}$ range from $10^{-8}$ to $10^{5} \mathrm{~Pa}$. Figure 6 shows $E_{\mathrm{f}}$ and $\varepsilon_{\mathrm{F}}$ for the cases of $E_{\mathrm{g}} / E_{\mathrm{g}}^{\mathrm{B}}=1.0$ and 0.6. As seen in Fig. 6 (b), for 
the case of $E_{\mathrm{g}} / E_{\mathrm{g}}^{\mathrm{B}}=1.0, V_{\mathrm{Al}}^{\prime \prime \prime}$ is more energetically favorable over the whole range of $P_{\mathrm{O}_{2}}$, but for the case of $E_{\mathrm{g}} / E_{\mathrm{g}}^{\mathrm{B}}=0.6$, the preference of $V_{\mathrm{Al}}^{\prime \prime \prime}$ or $V_{\mathrm{O}}^{\bullet \bullet}$ formation changes depending on $P_{\mathrm{O}_{2}}$. The switchover in the type of dominant vacancy is consistent with the results from oxygen permeation experiments. The dependence of $E_{\mathrm{f}}$ on $E_{\mathrm{g}}$ is shown in Fig. 7 (c) for both limits of $P_{\mathrm{O}_{2}}$. This figure indicates that the switchover appears somewhere in the range of $E_{\mathrm{g}} / E_{\mathrm{g}}^{\mathrm{B}}$ below 0.65 . The results suggest the importance of the narrowing of the band gap of a GB in connection with the switchover phenomenon because the band gap reduction of bulk crystals at high temperature is about 0.8 , as mentioned above.

The different behavior of Fermi levels in Figs. 6 (a), 7 (a), and 7 (b) provides some insight into the defect equilibrium. In Figs. 6 (a) and 7 (a), Fermi levels for the Schottky equilibrium, i.e., $2\left[V_{\mathrm{Al}}^{\prime \prime \prime}\right]=3\left[V_{\mathrm{O}}^{\mathbf{*}}\right]$, are also shown. The different dependence of $\varepsilon_{\mathrm{F}}$ on $P_{\mathrm{O}_{2}}$ for large and small band gaps are found in Fig. 6 (a). Comparison with the Schottky equilibrium indicates that the defect concentrations can be very different from those predicted from the most simple Schottky model over a wide range of $P_{\mathrm{O}_{2}}$, including around $1 \mathrm{~atm}$. This is also seen in Fig. 7 (a), where the Fermi energies are invariant and higher than those for Schottky equilibrium in the range of $E_{\mathrm{g}} / E_{\mathrm{g}}^{\mathrm{B}}>0.7$. This behavior shows that conduction electrons are sufficiently passive to have no effect on the equilibrium even at such a high temperature. In this range, the Fermi level is less than half the band gap, as seen in Fig. 7 (b), and relatively near to the valence band maximum. Thus, in the bulk-like region with a wide band gap, the defect equilibrium is determined by $\mathrm{Al}$ vacancies and holes, so the Schottky equilibrium is energetically unfavorable. As in 
Fig. 7 (b), when the band gap is decreased to the range of $E_{\mathrm{g}} / E_{\mathrm{g}}^{\mathrm{B}}<0.7, \varepsilon_{\mathrm{F}}$ lies at about the midpoint of the band gap and the defect formation energies are no longer constant, resulting in the change of the dominant vacancy. This is apparently caused by the participation of conduction electrons in the equilibrium between charged species according to Eq. (10). Because the increase in the number of electrons induces not only an enhancement of $V_{\mathrm{O}}^{\bullet \bullet}$ formation but also suppression of $V_{\mathrm{Al}}^{\prime \prime \prime}$ formation (via the decrease in number of holes), the behavior is relatively complex and seems to be independent of $\varepsilon_{\mathrm{F}}$ for the Schottky equilibrium as seen in Fig. 7 (a). At a constant $E_{\mathrm{g}}$ in the range of $0.55<E_{\mathrm{g}} / E_{\mathrm{g}}^{\mathrm{B}}<0.65$, when $P_{\mathrm{O}_{2}}$ increases from $10^{-8} \mathrm{~Pa}$ to $10^{5}$ $\mathrm{Pa}, \varepsilon_{\mathrm{F}}$ decreases by less than $0.5 \mathrm{eV}$. The change in $\varepsilon_{\mathrm{F}}$ is sensitive to large changes in the formation energies as a function of $P_{\mathrm{O}_{2}}$, as illustrated in Fig. 6.

Concentrations of charged species for the case of $E_{\mathrm{g}} / E_{\mathrm{g}}^{\mathrm{B}}=0.6$ and $T=1900 \mathrm{~K}$ are plotted in Fig. 8. This figure confirms the switchover expected from the formation energies of the vacancies, and the electronic carriers are found to be the dominant charge carriers. At high $P_{\mathrm{O}_{2}}$, holes are dominant and seem to enhance the formation of $V_{\mathrm{Al}}^{\prime \prime \prime}$; this tendency is consistent with the dominant reaction forming alumina (Eq. (2)) suggested by oxygen permeation experiments. In contrast, the enhanced formation of $V_{\mathrm{O}}^{\bullet \bullet}$ appears to be related to the electrons being the dominant carrier in accord with the reaction in Eq. (1). The transition from $p$-type conductivity at high $P_{\mathrm{O}_{2}}$ to $n$-type conductivity at low $P_{\mathrm{O}_{2}}$ mentioned by Heuer et al. [16] is, therefore, reproduced. Since the dominant carriers have a strong effect on the concentrations of vacancies, the effect of segregated dopants at GBs 
on the GB diffusion may be interpreted as resulting from the charge balance that also include the charge carriers from acceptors or donors. To probe this phenomenon further, the defect structures and charge states of the dopants at the GBs should be determined.

\section{Conclusion}

The switchover of the dominant defects in $\alpha-\mathrm{Al}_{2} \mathrm{O}_{3}$ as a function of $P_{\mathrm{O}_{2}}$ as suggested by oxygen permeation experiments were investigated by calculating the formation energies of charged oxygen and aluminum vacancies using DFT, where the HSE06 hybrid-type functional was used to obtain more reliable band gaps than those obtained using conventional LDA and GGA functionals. Fermi levels were determined assuming charge neutrality between the charged vacancies, holes, and conduction electrons. Examination of the electronic structures of the GBs revealed that the band gap of GBs is reduced by around $60 \%$ compared to the bulk value, depending on the degree of interface coherence and/or disorder. Thus, the dependence of the formation energies on the band gap was also examined. It was shown that the formation energies and the Fermi levels exhibit different behavior for wide band gap and narrower band gap structures. For a wide band gap GB (greater than $70 \%$ compared to the bulk value) the aluminum vacancies and holes are dominant regardless of $P_{\mathrm{O}_{2}}$. A crossover in the formation energies of the two types of vacancies appears only when the band gap is reduced to below $\sim 65 \%$ and above $\sim 55 \%$ of that in the bulk. Our results suggest that the electronic structures relative to not only the vacancy formation but also electronic carriers which are predominant should be discussed to understand 
the behavior observed in oxygen permeation experiments in $\alpha-\mathrm{Al}_{2} \mathrm{O}_{3}$.

\section{Acknowledgements}

This work was partially supported by the Japan Science and Technology Agency (Advanced Low Carbon Technology Research and Development Program) and by a Grant-in-Aid for Scientific Research on Innovative Areas "Nano Informatics" (25106008) from JSPS.

\section{References}

\section{References}

[1] Prescott R, Graham MJ. Oxid Met 1992;38:233.

[2] Stott FH. High Temp Corros Prot Mater 1997;4:19.

[3] Kitaoka S, Matsudaira T, Wada M. Mater Trans 2009;50:1023.

[4] Wada M, Matsudaira T, Kitaoka S. J Ceram Soc Jpn 2011;119:832.

[5] Matsudaira T, Wada M, Saitoh T, Kitaoka S. Acta Mater 2010;58:1544.

[6] Matsudaira T, Wada M, Saitoh T, Kitaoka S. Acta Mater 2011;59:5440.

[7] Matsudaira T, Kitaoka S, Shibata N, Nakagawa T, Ikuhara Y. J Mater Sci 2011;46:4407.

[8] Matsudaira T, Wada M, Kitaoka S. J Am Ceram Soc 2013;96:3243.

[9] Prot D, Le Gall M, Lesage B, Huntz AM, Monty C. Philos Mag A 1996;73:935. 
343

344

345

346

347

[10] Messaoudi K, Huntz AM, Lesage B. Mater Sci Eng A 1998;247:248.

[11] Nakagawa T, Sakaguchi I, Shibata N, Matsunaga K, Mizoguchi T, Yamamoto T, Haneda H, Ikuhara Y. Acta Mater 2007;55:6627.

[12] Heuer AH. J Eur Ceram Soc 2008;28:1495.

[13] Nakagawa T, Nishimura H, Sakaguchi I, Shibata N, Matsunaga K, Yamamoto T, Ikuhara Y. Scr Mater 2011;65:544.

[14] Paladino AE, Kingery WD. J Chem Phys 1962;37:957.

[15] Le Gall M, Lesage B, Bernardini J. Philos Mag A 1994;70:761.

[16] Heuer AH, Hovis DB, Smialek JL, Gleeson B. J Am Ceram Soc 2011;94:s146.

[17] Matsunaga K, Tanaka T, Yamamoto T, Ikuhara Y. Phys Rev B 2003;68:085110.

[18] Hine NDM, Frensch K, Foulkes WMC, Finnis MW. Phys Rev B 2009;79:024112.

[19] Hine NDM, Haynes PD, Mostofi AA, Payne MC. J Chem Phys 2010;133:114111.

[20] Choi M, Janotti A,Van de Walle CG . J Appl Phys 2013;113:044501.

[21] Zhang SB, Northrup JE. Phys Rev Lett 1991;67:2339.

[22] Fabris S, Elsässer C. Phys Rev B 2001;64:245117. 
[23] Höche T, Kenway PR, Kleebe HJ, Finnis MW, Rühle M. J Phys Chem Solids 1994;55:1067.

[24] Nakamura K, Mizoguchi T, Shibata N, Matsunaga K, Yamamoto T, Ikuhara Y. Phys Rev B 2007;75:184109.

[25] Matsunaga K, Nishimura H, Muto H, Yamamoto T, Ikuhara Y. Appl Phys Lett 2003;82:1179.

[26] Buban JP, Matsunaga K, Chen J, Shibata N, Ching WY, Yamamoto T, Ikuhara Y. Science 2006;331:212.

[27] Linstrom PJ, Mallard WG (Eds.). NIST Chemistry WebBook, NIST Standard Reference Database Number 69, National Institute of Standards and Technology, http://webbook.nist.gov, (retrieved July 7, 2013).

[28] Blöchl PE. Phys Rev B 1994;50:17953.

[29] Kresse G, Joubert D. Phys Rev B 1999;59:1758.

[30] Perdew JP, Burke K, Ernzerhof M. Phys Rev Lett 1996;77:3865.

[31] Krukau AV, Vydrov OA, Izmaylov AF, Scuseria GE. J Chem Phys 2006;125:224106.

[32] Monkhorst HJ, Pack JD. Phys Rev B 1976;13:5188.

[33] d'Amour H, Schiferl D, Denner W, Schulz H, Holzapfel. J Appl Phys 1978;49:4411.

[34] French RH. J Am Ceram Soc 1990;73:477. 
383 [35] Kuznetsov AI, Abramov VN, Mürk VV, Namozov BP. Sov Phys Solid $384 \quad$ State 1991;33:1126.

385 [36] Körner W, Bristowe PD, Elsässer C. Phys. Rev. B 2011;84:045305.

386 [37] Momida H, Nigo S, Kido G, Ohno T. Appl Phys Lett 2011;98:042102.

387 [38] Müllejans H, French RH. J Phys D 1996;29:1751.

388 [39] Mo SD, Ching WY, French RH. J Am Ceram Soc 1996;79:627.

389 [40] French RH, Jones DJ, Loughin S. J Am Ceram Soc 1994;77:412.

390 [41] Allen PB, Cardona M. Phys Rev B 1983;27:4760. 

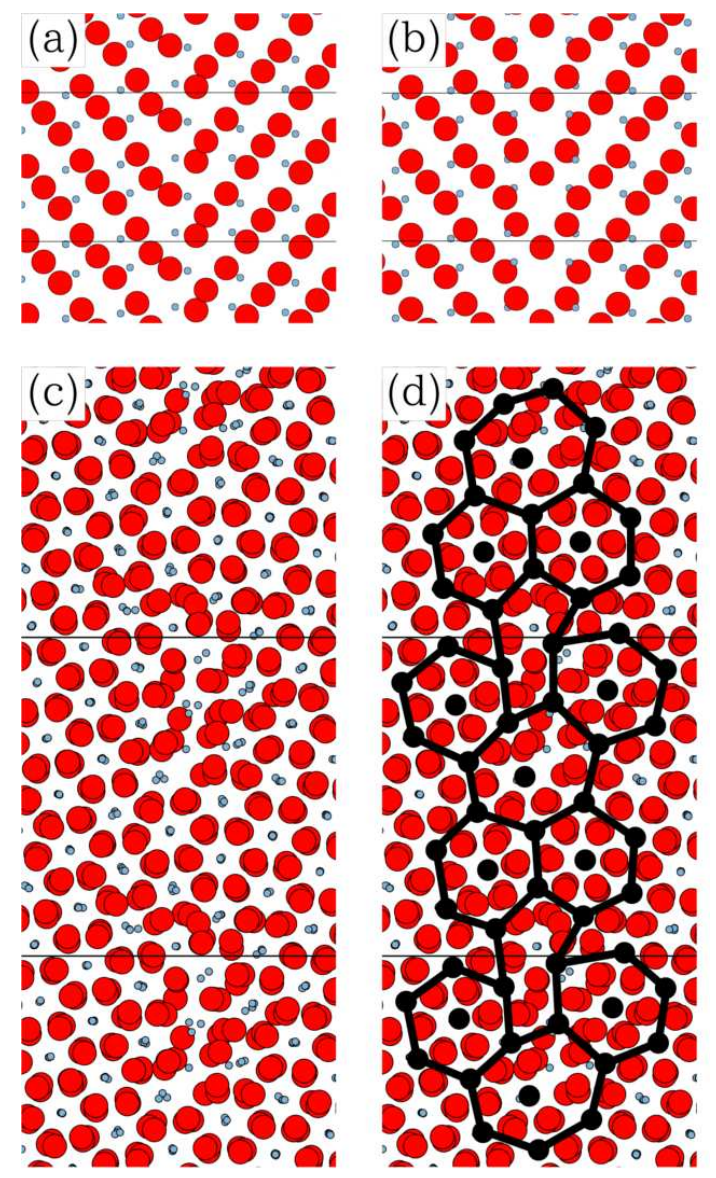

Figure 1: Atomic structures of $\alpha-\mathrm{Al}_{2} \mathrm{O}_{3}$ grain boundary models: (a) $\Sigma 13 \mathrm{G}(\mathrm{Al})$, (b) $\Sigma 13$ $\mathrm{G}(\mathrm{O})$ viewed down the $[10 \overline{1} 0]$ axis, and (c) $\Sigma 31$ viewed down the [0001] axis. The black circles in panel (d) indicate the network of aluminum atoms observed by transmission electron microscopy overlaid on the $\Sigma 31$ GB model in (c) $[25,26]$. Large red and small blue spheres represent oxygen and aluminum atoms, respectively. 

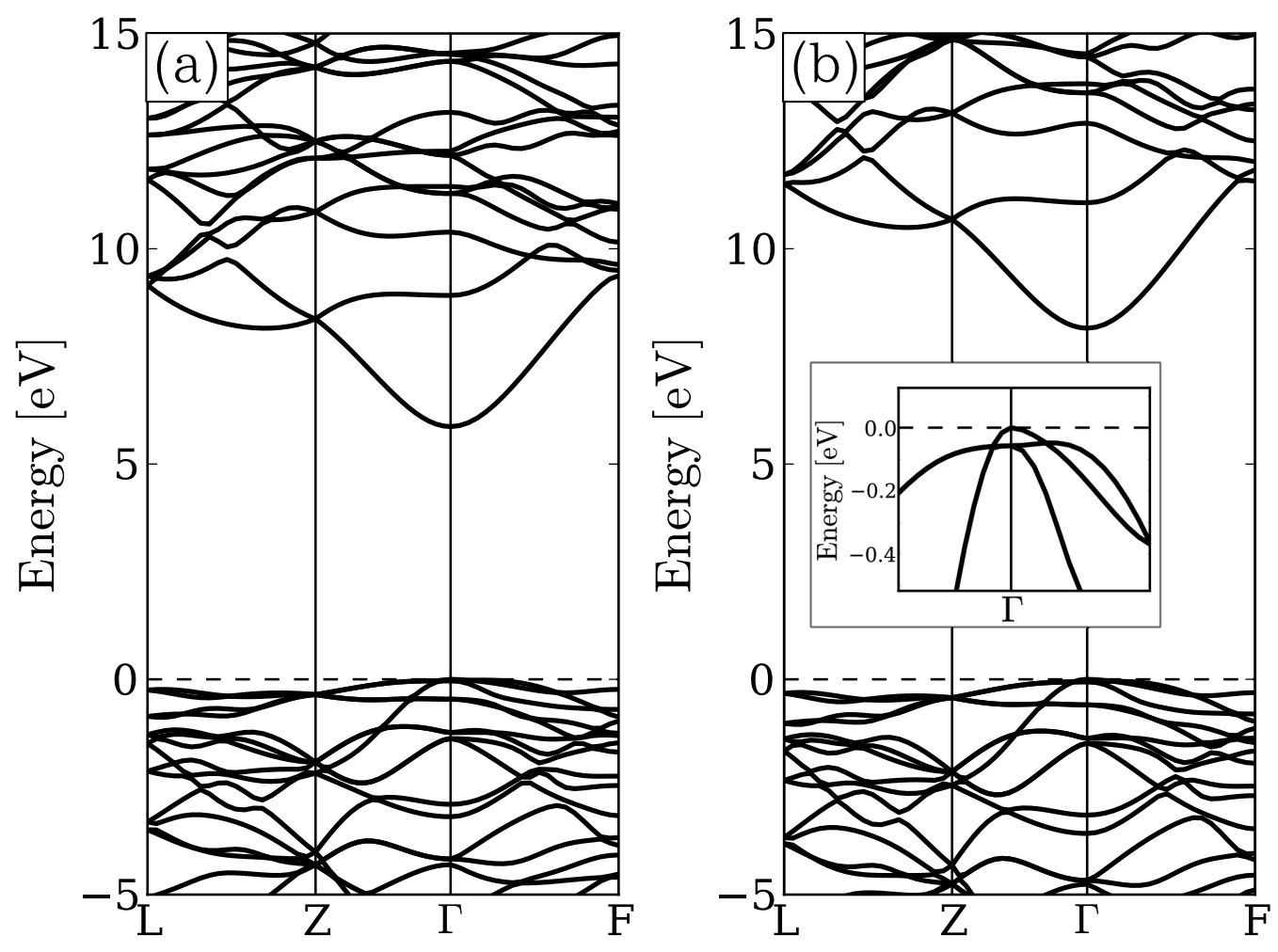

Figure 2: Electronic band structures of single-crystal $\alpha-\mathrm{Al}_{2} \mathrm{O}_{3}$ from (a) GGA and (b) HSE06 calculations. Inset in (b) shows a magnified view of bands around the valence band maximum. 


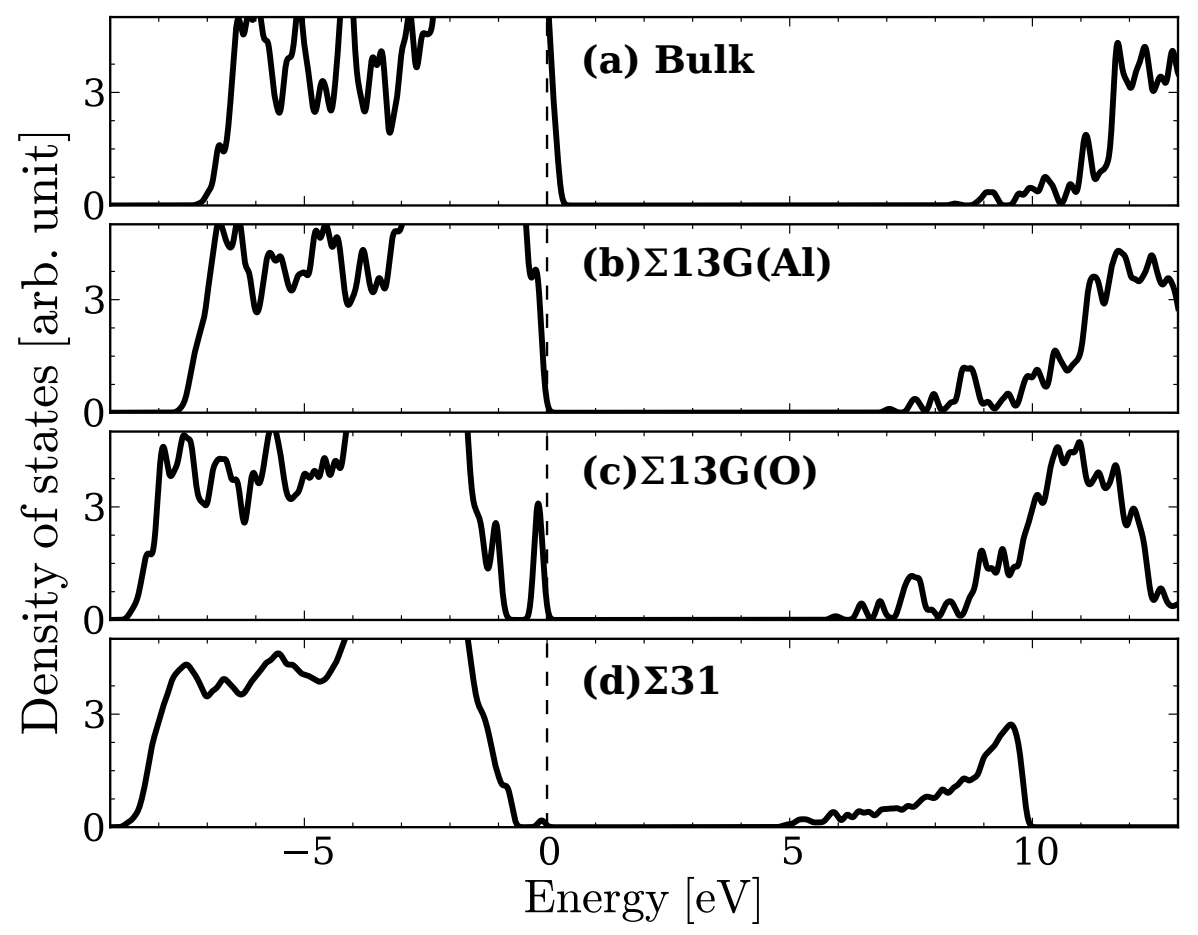

Figure 3: Density of states of (a) bulk crystal, (b) $\Sigma 13 \mathrm{G}(\mathrm{Al})$, (c) $\Sigma 13 \mathrm{G}(\mathrm{O})$, and (d) $\Sigma 31$ grain boundaries of $\alpha-\mathrm{Al}_{2} \mathrm{O}_{3}$ as a function of energy with respect to the highest occupied level using the HSE06 functional. 


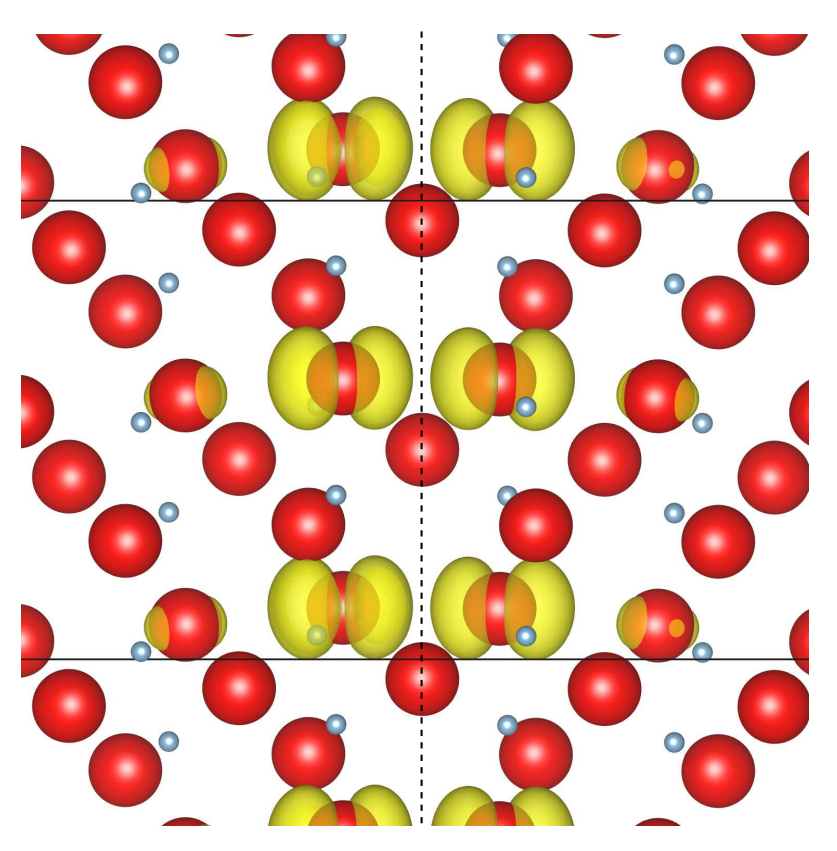

Figure 4: Partial charge density of the valence band maximum of a $\Sigma 13 \mathrm{G}(\mathrm{O})$ grain boundary shown in Fig.3 (c). The isosurface corresponds to 0.05 e/ $\AA^{3}$. 


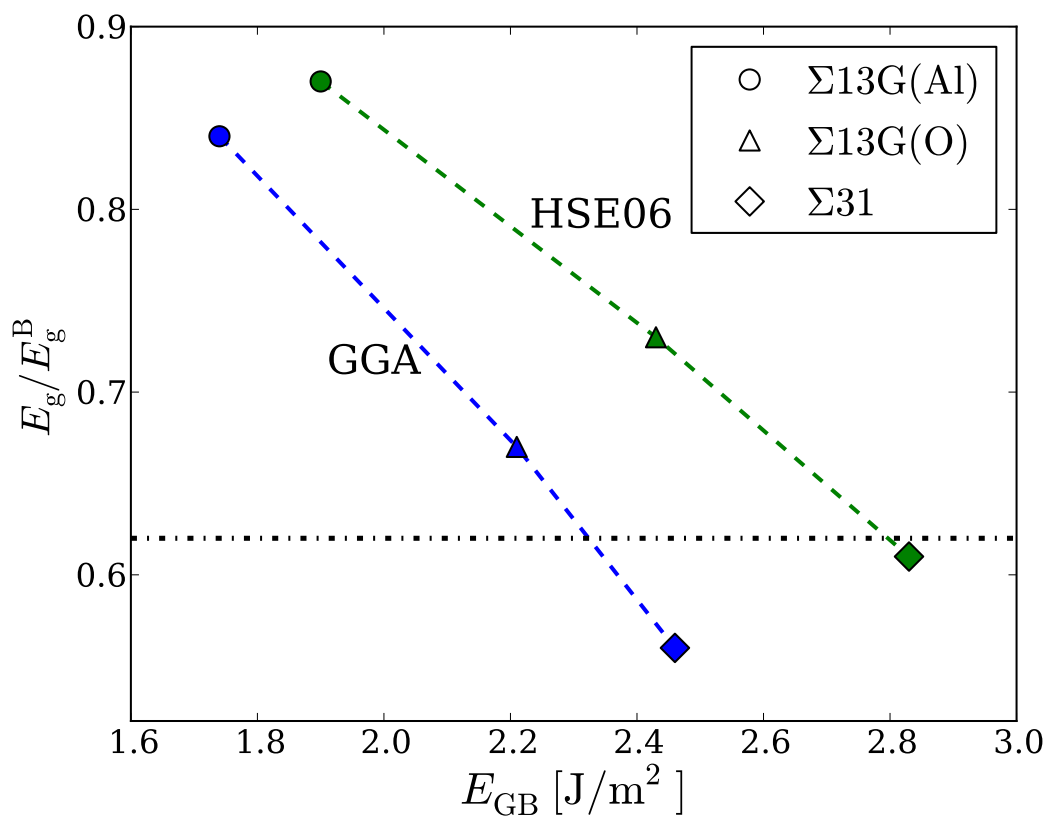

Figure 5: Band gap of $\alpha-\mathrm{Al}_{2} \mathrm{O}_{3}$ grain boundaries relative to the bulk gap as a function of grain boundary energy. The horizontal dotted line indicates the calculated value for amorphous $\mathrm{Al}_{2} \mathrm{O}_{3}[37]$. 

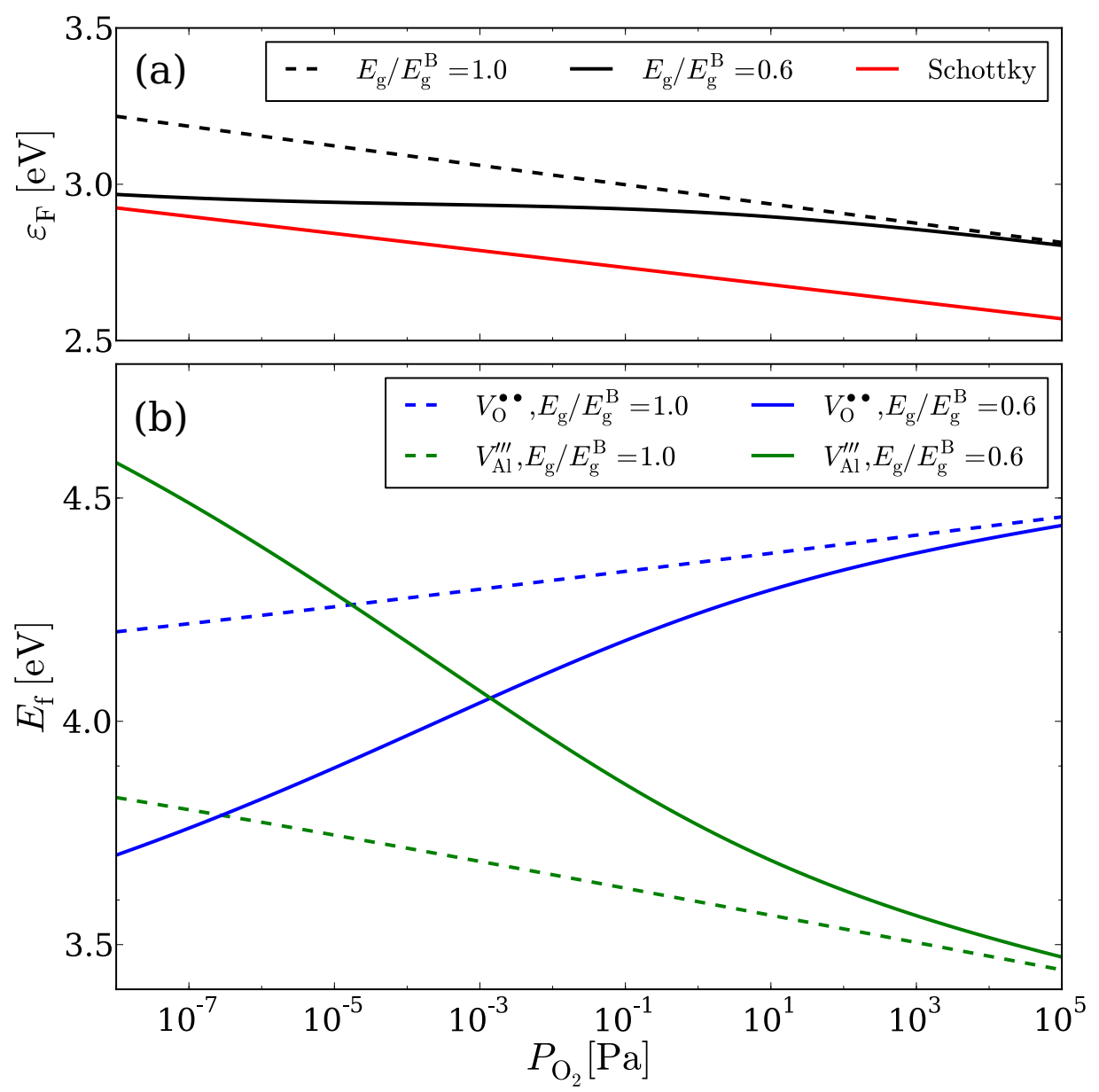

Figure 6: (a) Fermi levels and (b) formation energies of oxygen and aluminum vacancies at $T=1900 \mathrm{~K}$ as a function of oxygen partial pressure for the case of a relative band gap of 1.0 and 0.6 compared to the bulk value. In panel (a), Fermi levels for Schottky equilibrium are also shown. 


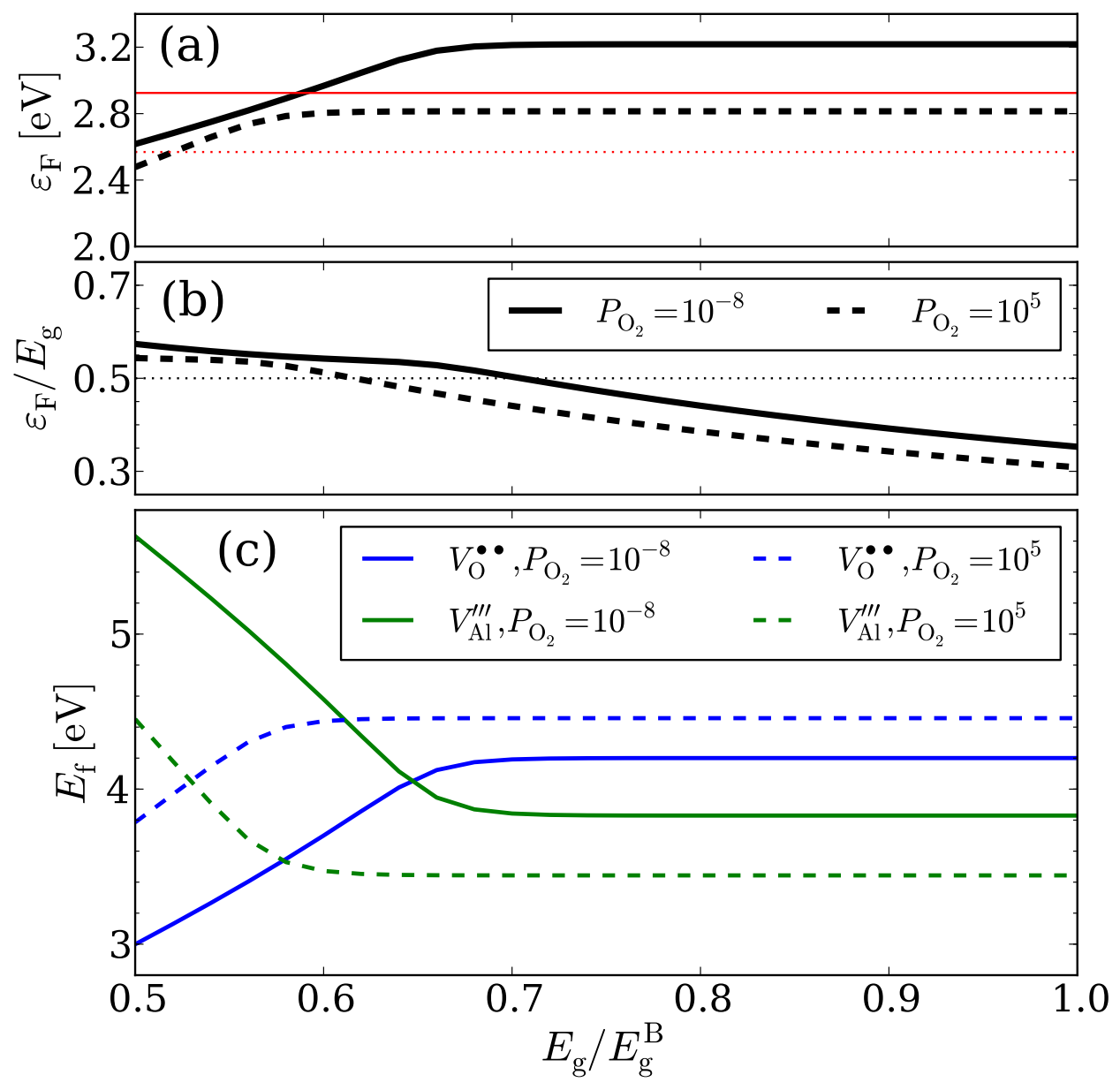

Figure 7: (a) Fermi levels, (b) Fermi levels divided by band gap, and (c) formation energies of oxygen and aluminum vacancies at $T=1900 \mathrm{~K}$ as a function of the relative band gap compared to the bulk value for the case of oxygen partial pressure $10^{-8} \mathrm{~Pa}$ and $10^{5} \mathrm{~Pa}$. In panel (a), Fermi levels for Schottky equilibrium are also shown for $10^{-8} \mathrm{~Pa}$ (thin red solid line) and $10^{5} \mathrm{~Pa}$ (thin red dotted line). In panel (b), the horizontal dotted line indicates half the band gap. 


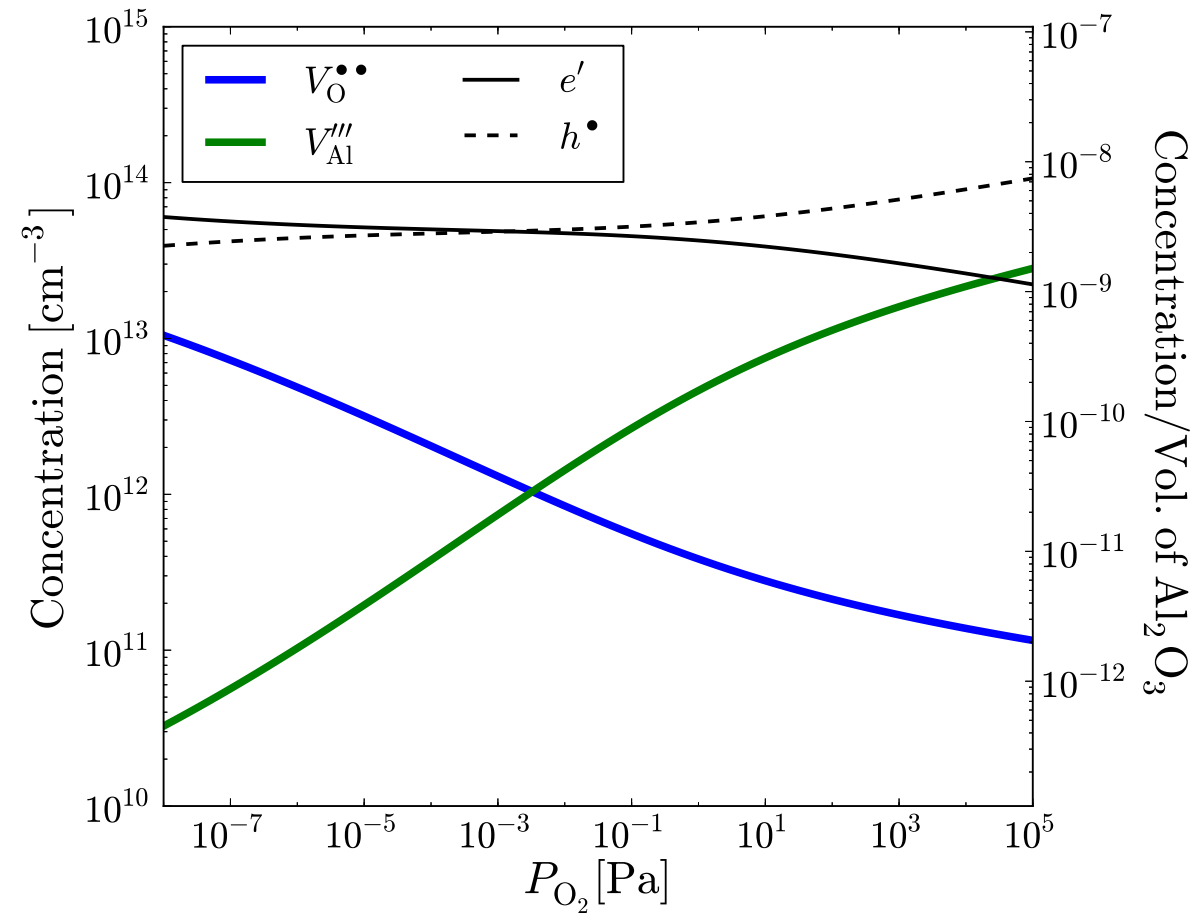

Figure 8: Concentration of oxygen and aluminum vacancies, holes, and electrons in the case of $E_{\mathrm{g}} / E_{\mathrm{g}}^{\mathrm{B}}=0.6$ and $T=1900 \mathrm{~K}$. The right vertical scale shows normalized concentration by the volume of bulk $\mathrm{Al}_{2} \mathrm{O}_{3}$ per formula unit. 